\title{
How to Publish Good Nuclear Medicine and Molecular Imaging Scientific Papers?
}

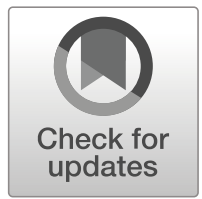

\section{E. Edmund Kim ${ }^{1,2}$}

Published online: 16 May 2020

(C) Korean Society of Nuclear Medicine 2020

Generally speaking, it is not easy to publish scientific papers in reputational journals with high impact factors due to low acceptance rates. Therefore, good science as well as good writing cannot be overemphasized. Nuclear medicine and molecular imaging is naturally research-oriented specialty of medicine. Its new techniques, methods, or radiopharmaceutical agents should be timely published in the major imaging and/or clinical journals to disseminate valuable and useful information. Rejection of scientific papers by the editors of good journals is related to poor science and writing as well as irrelevant topic, insufficient original work, methodology without rigor, poor analysis of results, and perhaps low acceptance rate. With my vast personal experiences as editor of the Current Medical Imaging as well as associate editor of the Journals of Nuclear Medicine and Radiology for many years, I would like to express my way to think about the nuclear medicine and molecular imaging scientific papers and help my trainees as well as junior faculties for their successful publication.

The authorship credit is obtained by substantial contribution to conception, design, acquisition, analysis or interpretation of data, or drafting of the article, or critical revision for important intellectual content. Agreement should be accountable for all aspects of the work in ensuring that questions related to the accuracy or integrity of any part of the article are appropriately investigated and resolved. The first author should be a main executor of the described work or main writer, and the last author is typically the senior researcher or supervisor. Multiple authorships may indicate a good responsible team work. Repetitive publication of the same

\section{E. Edmund Kim}

edmundek@uci.edu

1 University of California at Irvine, Irvine, USA

2 Seoul National University and Kyunghee University, Seoul, South Korea methods, experiments, or data is considered plagiarism which makes unethical and bad consequences to your reputation.

A scientific paper is a written report describing original research results with the format defined by the traditional and editorial practices as well as ethics, and also interplaying printing and publishing services. It usually includes research, review, and educational and case description papers as well as papers on developed systems or applications. The typical research paper deals with new concepts, problems, approaches to known problems, algorithms, devices, and experiments. The results need to be compared with the state of the art. Quality should be preferred over quantity. The review paper is organized and structured with descriptions of a cutting-edge research theme. The important information may be scattered across different sources and hard to find elsewhere. Therefore, it should summarize, evaluate, or synthesize already published information and provide sources of new ideas. The paper on developed system or application needs to describe problems to solve, developmental difficulties, and implementation choices. It is also necessary to compare the performance, usability, and features of the system with others.

Why do we want to publish scientific papers? It may be helpful for your academic or scientific career with the creation of knowledge which is more than on the transmission. Others can benefit from your contribution to understanding the world. It is also useful to make records of your valuable works and challenges.

What can you publish? A new idea such as the first solution to an impacting problem is the best to publish. You also may publish results of experiments, general or specific problems, proof of the impossibility of solving a problem, better solution to a known problem, knowledge gaps, multidisciplinary ideas, and integration of knowledge, trends in recent, cutting-edge areas.

At present time, topics related to theranostics and molecular imaging as well as imaging immunotherapy or immune metabolic imaging seem to have relatively high acceptance rates in the Journal of Nuclear Medicine. Imaging fibrosis, 
radionanomedicine, and application of artificial intelligence or machine learning in clinical imaging are growing and promising in the field of nuclear medicine and molecular imaging.

What is a good scientific paper? It starts with a good research idea. The original idea must demonstrate scientific, economic, and social impact of the studied problem. It also should have sound methodology and good data analysis with good critical coverage of related literature. The clarity of presentation and thought-provoking discussion is essential as the way of communication and dissemination.

The following questions are important for good scientific papers: Is the research new? Is the research significant to the field of research? Does it outline the current knowledge of the problem and existing solution? Does it point out the contribution to the problem solution? Does it present any preliminary ideas, the proposed approach, and results achieved so far? Does it sketch the methodology? Does it state how and by whom the results are evaluated, compared with existing approaches, or applied?

What is a good scientific writing? Nothing works better than reading journals and books a lot to improve your writing skills. It is better to publish your paper preferably in English so that your research is accessible worldwide. Good scientific writing is accurate (avoid vague and ambiguous language), objective (supported by appropriate evidence), impartial (avoid assumptions and unproven statements), clear (avoid unnecessary detail), simple (avoid vague and complicated sentences), and structured logically.

How to write good scientific papers? You have to think carefully about your topic and ensure that it is sufficiently focused. You also need to devote time to planning and stick to your plan, and then write a detailed research proposal and discuss with your colleagues as well as supervisor. You have to be organized and take detailed notes when you are undertaking your literature survey and data collection. You must move positively into writing-up your research and allocate enough time to reviewing and editing your writing.

To make your writing accurate, concise, and clear, you should consider carefully the English words that you use and the ways in which you use them. Objective language is impartial and states a fact or process, while subjective language is open to question or interpretation. In most scientific writing, you will need to use scientific or technical terms to be clear and unambiguous. However, it is better to use such terms only when you need to do so. Scientific writing frequently uses the past tense, particularly when the main focus of the writing is to describe experiments or observations that took place prior to the time of writing. However, the past tense may not be appropriate for everything that you write and sometimes you will need to combine different tenses. Appropriate use of past, present, and future tenses can contribute to a clear and unambiguous writing style. To write clearly and succinctly, it is better to write at a level that is appropriate for your audience, use active voice, the first person ("I think" instead of "It is thought"), short words, verbs instead of abstract nouns, and strong verbs instead of "to be".

For beginners, it is better to find a role model and then follow the model of a good paper of the kind you are writing in your research field. You also have to write simple, short unstructured notes or ideas that come to your mind on the day you start your research. It is useful to search and read good and updated bibliographic database a bit every day.

For clearer and more readable paper, you have to try to compress your paper and repeat until you cannot compress any longer but keeping coherence and completeness. You will focus on the relevant stuff, and you will find out that extra detail was not so important.

Good writing is iterative, and you should not expect a perfect manuscript at first attempt. Poor presentation will frustrate the reviewers, and they will get a negative predisposition and will not read your paper properly.

To organize good scientific papers, you have to prepare the figures and tables, write the methods, the results, and the discussion before writing the introduction since you cannot objectively demonstrate the scientific significance of your work in the introduction if the discussion is insufficient. You also have to write clear conclusion, compelling introduction, and the abstract, and compose a concise and descriptive title and select keywords for indexing.

The title describes the contents of the paper with the fewest possible words. Only a few generally read the entire paper, but the title is read by many people. Therefore, all words in the title should be chosen with care. Too short a title is not helpful to the reader, but too long a title can sometimes be even less meaningful. You should use specific terms rather than general and avoid abbreviations and jargon in the title. The abstract is often the most important part of the paper since most readers only read that. Readers also use the abstract to decide whether or not to read and cite the paper. The abstract is a brief description of the whole paper so that diagonal readers understand it without reading the other part of the manuscript. Therefore, it needs to focus on what is new and on key information on the purpose, methodology, results, findings, and conclusion as well as recommendations. Acronyms should be avoided. If they must be used, their definition should be repeated in the main text. It is better to the abstract last before the completion of the paper.

In the introduction, you have to describe briefly and clearly why you are writing the paper. The introduction supplies sufficient background information and presents the nature and scope of the problem investigated. It also states the method and the principle results of the experiment. Therefore, careful writing of the materials and methods must be made for your results to be of scientific merit and reproducible. You should state exact technical specifications, quantities, source, or method of preparation. You also need to describe equipment 
used and provide illustrations where relevant. It is important to discuss statistical methods only if unusual or advanced. You need to give an overall description of the experiments in the results and present the data you found. You have to order your results logically, starting with the most important ones. Short and sweet factual statements supported by evidence should be presented. You have to discuss variables only if they made a positive or negative effect and also use meaningful statistics. It is not necessary to describe every step of your statistical analyses. You must refer in the text to each figure or table you include in your paper. A figure or graph is needed only when the data lend themselves to a good visual representation or support statistical analysis. The quality of figures and tables is fundamental and most important. Tables generally should report summary-level data. The results should be sequential, linear, rigorous, and explicit or specific. The hardest section to write is the discussion, and you are trying to bring out the true meaning of your data without being too long. You should not use words to conceal your facts or reasoning, and also, you should not repeat your results. You should present principle, relationships, and generalizations shown by the results and show how your results agree or disagree with previously published works. You must discuss the theoretical implications of your work as well as practical applications, and also summarize your evidence of each conclusion and the significance of the results. It is good to make a list of the keywords that reflect the described work with the minimum number of words. Sometimes the title may contain the conclusion of the paper. It is better to rewrite the title in the final version of your paper. Finally, you have to carefully review your references with good credibility, particularly years, in the desired format. Too many references may indicate lack of capacity to discern essential from accessory. Reference list contains only materials that are cited in your document, and bibliography includes all sources consulted for background or further reading.

It would be good and helpful for you to make your draft paper and then present it in your departmental conference or seminar as well as national or international conferences to receive valuable criticisms or comments for the final completion of your paper.

\section{Compliance with Ethical Standards}

Conflict of Interest Edmund Kim declares that he has no conflict of interest. There is no source of funding.

Ethical Approval This article does not contain any studies with human participants performed by any of the authors.

Informed Consent Not applicable.

\section{Further Reading}

1. Mack CA. How to write a good scientific paper: citations. Journal of Micro/Nanolithography, MEMS, and MOEMS. 2012;11:030101.

2. The write stuff: Nature (CAREERS), pp. 129-130, 2018

Publisher's Note Springer Nature remains neutral with regard to jurisdictional claims in published maps and institutional affiliations. 